9.

\title{
DEMONSTRATION OF PASCAL'S THEOREM.
}

[From the Cambridge Mathematical Journal, vol. Iv. (1843), pp. 18-20.]

Lemma 1. Let $U=A x+B y+C z=0$ be the equation to a plane passing through a given point taken for the origin, and consider the planes

$$
U_{1}=0, \quad U_{2}=0, \quad U_{3}=0, \quad U_{4}=0, \quad U_{5}=0, \quad U_{6}=0 ;
$$

the condition which expresses that the intersections of the planes (1) and (2), (3) and (4), (5) and (6) lie in the same plane, may be written down under the form

$$
\left|\begin{array}{cccccc}
A_{1}, & A_{2}, & A_{3}, & A_{4} & . & . \\
B_{1}, & B_{2}, & B_{3}, & B_{4} & . & . \\
C_{1}, & C_{2}, & C_{3}, & C_{4} & . & . \\
. & . & A_{3}, & A_{4}, & A_{5}, & A_{6} \\
. & . & B_{3}, & B_{4}, & B_{5}, & B_{6} \\
. & . & C_{3}, & C_{4}, & C_{5}, & C_{6}
\end{array}\right|=0 .
$$

Lemma 2. Representing the determinants

$$
\left|\begin{array}{lll}
x_{1}, & y_{1}, & z_{1} \\
x_{2}, & y_{2}, & z_{2} \\
x_{3}, & y_{3}, & z_{3}
\end{array}\right| \& c .
$$

by the abbreviated notation $\overline{123}$, \&c.; the following equation is identically true:

$$
\overline{345} \cdot \overline{126}-\overline{346} \cdot \overline{125}+\overline{356} \cdot \overline{124}-\overline{456} \cdot \overline{123}=0 .
$$


This is an immediate consequence of the equations

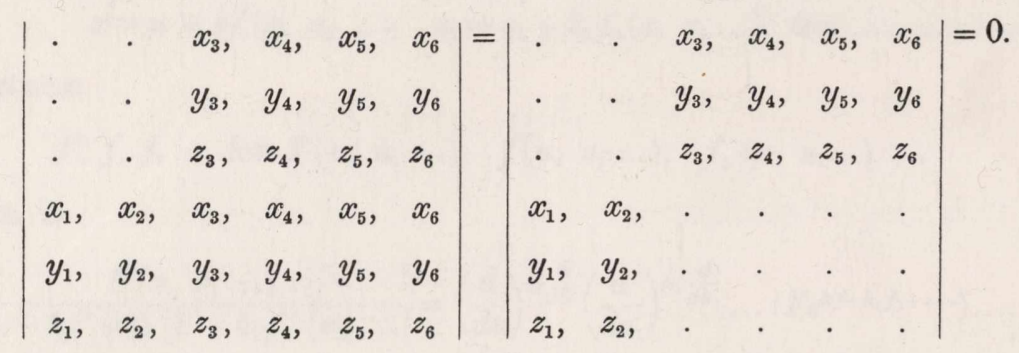

Consider now the points $1,2,3,4,5,6$, the coordinates of these being respectively $x_{1}, y_{1}, z_{1} \ldots \ldots x_{6}, y_{6}, z_{6}$. I represent, for shortness, the equation to the plane passing through the origin and the points 1,2 , which may be called the plane $\overline{12}$, in the form

$$
x \overline{12}_{x}+y \overline{12}_{y}+z \overline{12}_{z}=0
$$

consequently the symbols $\overline{12}_{x}, \overline{12}_{y}, \overline{12}_{z}$ denote respectively $y_{1} z_{2}-y_{2} z_{1}, z_{1} x_{2}-z_{2} x_{1}, x_{1} y_{2}-x_{2} y_{1}$, and similarly for the planes $\overline{13}, \&$ c. If now the intersections of $\overline{12}$ and $\overline{45}, \overline{23}$ and $\overline{56}$, $\overline{34}$ and $\overline{61}$ lie in the same plane, we must have, by Lemma (1), the equation

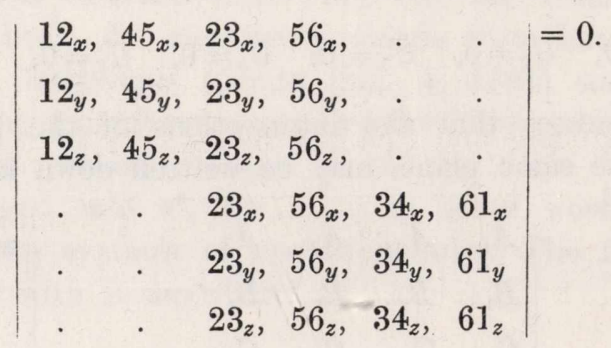

Multiplying the two sides of this equation by the two sides respectively of the equation

$$
\left|\begin{array}{cccccc|}
x_{6}, & x_{1}, & x_{2}, & \cdot & . & \cdot \\
y_{6}, & y_{1}, & y_{2}, & . & . & \cdot \\
z_{6}, & z_{1}, & z_{2}, & . & . & . \\
\cdot & \cdot & \cdot & x_{3}, & x_{4}, & x_{5} \\
\cdot & \cdot & \cdot & y_{3}, & y_{4}, & y_{5} \\
\cdot & . & . & z_{3}, & z_{4}, & z_{5}
\end{array}\right|
$$

and observing the equations

$$
x_{6} \overline{12}_{x}+y_{6} \overline{12}_{y}+z_{6} \overline{12}_{z}=\overline{612}, \quad \overline{112}=0, \& c .
$$


this becomes

$$
\left|\begin{array}{cccccc}
612 & . & . & \cdot & . & \cdot \\
645, & 145, & 245, & \cdot & . & . \\
623, & 123, & \cdot & \cdot & 423, & 523 \\
\cdot & 156, & 256, & 356, & 456, & . \\
\cdot & \cdot & \cdot & . & . & 534 \\
. & . & . & 361, & 461, & 561
\end{array}\right|=0
$$

reducible to

$\overline{612} \overline{534}\left|\begin{array}{cccc}\overline{145}, & \overline{245}, & . & . \\ 123, & . & . & 423 \\ 156, & 256, & 356, & 456 \\ . & . & 361, & 461\end{array}\right|=0$

or, omitting the factor $\overline{612} \cdot \overline{534}$ and expanding,

$\overline{145} \cdot \overline{256} \cdot \overline{423} \cdot \overline{361}+\overline{245} \cdot \overline{123} \cdot \overline{456} \cdot \overline{361}-\overline{245} \cdot \overline{123} \cdot \overline{356} \cdot \overline{461}-\overline{245} \cdot \overline{156} \cdot \overline{423} \cdot \overline{361}=0$.

Considering for instance $x_{6}, y_{6}, z_{6}$ as variable, this equation expresses evidently that the point 6 lies in a cone of the second order having the origin for its vertex, and the equation is evidently satisfied by writing $x_{6}, y_{6}, z_{6}=x_{1}, y_{1}, z_{1}$, or $x_{3}, y_{3}, z_{3}$, or $x_{4}, y_{4}, z_{4}$, or $x_{5}, y_{5}, z_{5}$, and thus the cone passes through the points $1,3,4,5$. For $x_{6}, y_{6}, z_{6}=x_{2}, y_{2}, z_{2}$, the equation becomes, reducing and dividing by $\overline{245} \cdot \overline{123}$,

$$
\overline{452} \cdot \overline{321}-\overline{352} \cdot \overline{421}+\overline{152} \cdot \overline{423}=0 \text {, }
$$

which is deducible from Lemma (2), by writing $x_{6}, y_{6}, z_{6}=x_{2}, y_{2}, z_{2}$, and is therefore identically true. Hence the cone passes through the point (2), and therefore the points $1,2,3,4,5,6$ lie in the same cone of the second order, which is Pascal's Theorem. I have demonstrated it in the cone, for the sake of symmetry; but by writing throughout unity instead of $z$, the above applies directly to the case of the theorem in the plane.

The demonstration of Chasles' form of Pascal's Theorem (viz. that the anharmonic relation of the planes $\overline{61}, \overline{62}, \overline{63}, \overline{64}$ is the same with that of $\overline{51}, \overline{52}, \overline{53}, \overline{54}$ ), is very much simpler; but as it would require some preparatory information with reference to the analytical definition of the similarity of anharmonic relation, I must defer it to another opportunity. 